\title{
Editorial
}

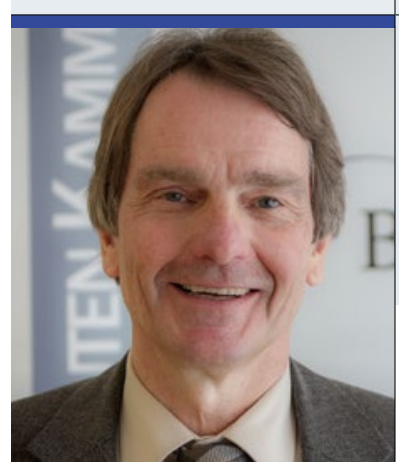

"Die Bundespsychotherapeutenkammer wird sich am

Aktionsplan für eine bessere ambulante Versorgung von

Patienten mit chronischen Schmerzen beteiligen."

\section{Schmerzpatienten gemeinsam besser versorgen}

S chmerztherapie ist interdisziplinär und hat ein weites Spektrum. Dies spiegelt sich erneut in den Beträgen dieser Ausgabe der „Schmerzmedizin" wider, in der ein breites Themenfeld von Schmerztherapie in der Palliativmedizin bis hin zur multimodalen Schmerztherapie von Migranten, die Krieg und Vertreibung erlebt haben, behandelt wird.

Die großen gesellschaftlichen Herausforderungen, vor denen wir aktuell in Deutschland stehen, verlangen in der Gesundheitsversorgung im Allgemeinen und in der Versorgung von Flüchtlingen und Migranten im Besonderen die Zusammenarbeit unterschiedlicher Berufsgruppen. Diese Kooperation setzt die Fähigkeit und die Bereitschaft zur Zusammenarbeit voraus. Als Vertreter einer der an der Schmerztherapie beteiligten Professionen, der Psychotherapeuten, möchte ich unseren klaren Willen zur Zusammenarbeit zum Ausdruck bringen.

Doch der gute Wille allein reicht für eine gelingende Versorgung nicht aus. Auch wenn die Bedeutung psychischer Faktoren für die Schmerzentstehung, -wahrnehmung und -bewältigung heute unumstritten ist und schmerzpsychologische Angebote ein fester Bestandteil der multimodalen Behandlung in vielen Schmerzzentren geworden sind, gibt es aus unserer Sicht noch viel zu tun.

Der Schwerpunkt schmerzpsychotherapeutischer Tätigkeit liegt derzeit im stationären und teilstationären Bereich. Allerdings sind insgesamt immer noch zu wenige Psychotherapeuten in Schmerzentren beschäftigt. Vor allem gibt es aber Defizite in der ambulanten schmerztherapeutischen Versorgung. Dabei sollten sich im ambulanten Bereich wie in den spezialisierten Schmerzeinrichtungen bereits üblich - interdisziplinäre Schmerzteams etablieren, in denen Hausärzte, Schmerzmediziner, Psychotherapeuten und Physiotherapeuten kooperieren und eng zusammenarbeiten. Die Bundespsychotherapeutenkammer wird sich deshalb am ge- meinsamen Aktionsplan der Deutschen Gesellschaft für Schmerzmedizin und der Deutschen Schmerzliga zur Entwicklung von Netzstrukturen für eine bessere ambulante Versorgung von Patienten mit chronischen Schmerzen beteiligen.

Die interdisziplinäre Zusammenarbeit ist eine der Herausforderungen, der sich ein auf dem Gebiet der Schmerztherapie arbeitender Psychologischer Psychotherapeut oder Kinder- und Jugendlichenpsychotherapeut stellen muss. Aber auch darüber hinaus setzt die Behandlung von Schmerzpatienten ein umfangreiches Wissen voraus. Vertiefte Kenntnisse über biologische, psychologische und soziale Grundlagen des Schmerzgeschehens, Mechanismen der Schmerzentstehung und -chronifizierung sowie der spezifischen Diagnostik und Behandlung bei unterschiedlichen Schmerzsyndromen sind Voraussetzungen für eine spezifische Schmerzpsychotherapie. Kenntnisse, die in der Ausbildung zum Psychotherapeuten noch nicht ausreichend vermittelt werden. Daher versuchen wir, die Schmerzpsychotherapie auch innerhalb unseres Fachs als einen wichtigen Bereich zu fördern, für den sich Psychotherapeuten nach der Ausbildung weiter qualifizieren können, um den Patienten eine bessere Versorgung - auch im ambulanten Bereich - zu bieten.

Den Herausforderungen der interdisziplinären Versorgung werden sich Psychotherapeuten darüber hinaus vor allem dann stellen, wenn sie positive Erfahrungen in der Kooperation mit den anderen Berufsangehörigen gemacht haben. Wenn uns das gelingt, bin ich zuversichtlich, dass wir mit vereinten „interdisziplinären“ Anstrengungen die Versorgung von Schmerzpatienten in Deutschland weiter verbessern werden.

Ihr

Dietrich Munz 